\title{
The use of an occlusive penile clamp during filling cystometry in men with symptoms of stress urinary incontinence
}

\author{
Ala'a Sharaf ${ }^{1}$, Mandy Fader ${ }^{2}$, Margaret Macaulay ${ }^{2}$, Marcus J. Drake ${ }^{1}$
}

1. Bristol urological institute, Southmead hospital, Bristol BS10 5NB, United Kingdom

2. Faculty of Health Sciences, University of Southampton, University Road, Southampton, SO17 1BJ, UK

- Mr Ala'a Sharaf: Clinical Research Fellow in Functional Urology, Bristol urological institute, Southmead hospital, Bristol BS10 5NB, United Kingdom. Telephone 00447796056077.

- Professor Mandy Fader, Head of School, Professor of Continence Technology. School of Health Sciences, University of Southampton, University Road, Southampton, SO17 1BJ, UK. Telephone: 02380597840.

- Miss Margaret Macaulay, Senior Research Nurse. School of Health Sciences, University of Southampton, University Road, Southampton, SO17 1BJ, UK. Telephone: 0238059 7840 .

- Professor Marcus Drake, Professor of Physiological Urology, University of Bristol. Bristol Urological Institute, Southmead Hospital, Bristol BS10 5NB, United Kingdom. Telephone 01779505050

The work was performed at Southmead Hospital, Bristol, BS10 5NB, United Kingdom.

Word Count: 2302 


\begin{abstract}
Introduction

In severe post prostatectomy stress urinary incontinence (SUI), urodynamics may not identify crucial parameters because of inadequate bladder filling. This study describes evaluation of cystometry and pressure flow study (PFS) in men where severe SUI during attempted filling necessitated application of a penile clamp to allow filling to reach cystometric capacity.
\end{abstract}

\title{
Methods
}

We identified all men who had undergone prior radical prostatectomy from a database of patients attending for videourodynamic testing between 2012-2017. Symptom scores, bladder diary and free flow rate tests were retrieved. We evaluated the measurements of the subgroup of men with severe SUI for whom a Thomson-Walker compression clamp was utilised to enable full urodynamic evaluation.

\section{Results}

166 radical prostatectomy patients were identified. In 30 (18\%), severe SUI led to incomplete filling cystometry, i.e. failure to reach cystometric capacity. Following application of the penile compression clamp, it was possible to achieve further filling in each case. Applying the clamp did not alter vesical filling or impede pressure recording. These men had a lower maximum urethral closure pressure ( 31.6 vs $46.5 \mathrm{cmH} 20 ; p<0.001$ ), volume at strong desire to void (132 vs $242 \mathrm{mls} ; \mathrm{p}=0.003$ ) and cystometric capacity ( 226 with the clamp applied vs $310 \mathrm{mls} ; \mathrm{P}<0.001$ ) when compared to the overall post prostatectomy incontinence population. Flow rates during PFS were comparable, but detrusor pressure at maximum flow was lower in the clamp group (11 vs $22 \mathrm{cmH} 2 \mathrm{O} ; \mathrm{p}=0.009$ ). Vesicoureteric reflux (VUR) was not seen in conjunction with the penile clamp use.

\section{Conclusions}

This study shows that the use of a penile clamp during urodynamics for incontinent men who have had a radical prostatectomy can optimise the test by aiding additional bladder filling in selected patients. This allows for a clearer interpretation of cystometric capacity and ability to undertake PFS. The short-term use in this context is well tolerated and does not raise any safety concerns.

Keywords: Urodynamics, penile clamp, incontinence, radical prostatectomy, reflux. 


\section{The use of an occlusive penile clamp during filling cystometry in men with symptoms of stress urinary incontinence}

\section{Introduction}

Urodynamics (UDS) is undertaken to identify mechanisms contributing to lower urinary tract symptoms (LUTS) and incontinence. It usually follows conservative therapy, where a patient is considering interventional treatment such as surgery due to persistence of symptoms. For example, in men with stress urinary incontinence (SUI), notably after radical prostatectomy, UDS may be done after pelvic floor muscle exercises, when the individual may be considering artificial urinary sphincter (AUS) or male sling placement. UDS is undertaken to ascertain causes of symptoms and risk factors that could influence therapy choice ${ }^{1}$. Thus, in men considering outlet surgery to treat SUI, UDS aims to identify the cause of incontinence, and also to evaluate factors which could adversely affect surgery outcome, for example impaired detrusor compliance ${ }^{2}$, detrusor overactivity (DO) and detrusor underactivity ${ }^{3}$.

For most men with post prostatectomy incontinence (PPI), there is some residual sphincter function, so that bladder filling will reach cystometric capacity in urodynamic testing. Indeed, for some men it can be difficult to demonstrate the presence of incontinence ${ }^{4}$. However, in a minority of PPI cases, a contrasting situation can be present, in that the bladder outlet may have insufficient function to retain an adequate proportion of the instilled liquid for reliable recording of vesical pressure or for complete bladder filling. Consequently, the urodynamic test may be unable to identify crucial parameters essential for treatment recommendations and patient decision-making. For example, compliance calculation during the storage phase relies on accurate recording of both pressure and volume. DO detection requires pressure measurement, and may only become evident at higher volumes during filling cystometry. Furthermore, cystometric capacity (CC) is a valuable parameter to estimate, since it will potentially anticipate voiding frequency once sphincter function is restored. Though of lesser clinical importance for decision making, severe SUI will also preclude measurement of bladder volume at first desire to void (FDV), normal desire to void (NDV), and strong desire to void (SDV). During pressure flow studies (PFS), detrusor underactivity cannot reliably be detected if the bladder is not filled to an adequate volume.

In order to overcome the problem of leakage during filling cystometry becoming so severe that cystometric capacity is not reached, a penile compression device (clamp) may be used to allow for adequate bladder filling. A clamp is an external device placed on the penile shaft to occlude the urethra and stop the loss of urine. Penile clamps are designed to be used as a management option for SUI for some men. There is evidence that men find clamps effective at containing urinary incontinence, in particular for short periods of physical activity. However, U.K. National Institute of Health and Care Excellence (NICE) guidance advice is not to offer penile clamps to men with storage LUTS. 
In theory, a penile clamp could be used to facilitate undertaking of urodynamic testing, by preventing leakage during the filling phase, whilst being easy to remove at cystometric capacity when giving permission to void. There are no published reports of this method, but a similar approach has been used to enable performance of a urethrogram in radiology 5 .

Accordingly, we surmised that a penile clamp could be used as a component of urodynamic testing in men with severe SUI to enable adequate bladder filling and assessment of vesical pressure $\left(\mathrm{P}_{\mathrm{ves}}\right)$ and volume parameters. The premise was to commence filling cystometry in the standard way for all men with PPI, and for any man who developed excessive leakage which would have precluded completion of testing, to apply the penile clamp during the remainder of filling, removing it after cystometric capacity was reached to enable PFS to be done. The aims of this study were to investigate and report on:

i) the effect of penile clamp use on filling cystometry and pressure flow studies in men with severe PPI.

ii) the association between clamp use and vesico-ureteric reflux.

\section{Methods}

The study was conducted in a U.K. tertiary referral centre which performs about 1,000 urodynamic studies a year, in men and women. We retrospectively reviewed patient data from a database of 1,256 men, who underwent urodynamic testing between November 2012 and March 2017. Patients who had radical prostatectomy had to wait at least 12 months before attending for urodynamics to allow for the possibility of spontaneous resolution with pelvic floor exercises and time.

The process used for urodynamics and the data collected during this period were as follows: a detailed history and demographic details were taken. International Consultation on Incontinence Questionnaire (ICIQ) on Male LUTS (ICIQ-MLUTS) and ICIQ Bladder Diary ${ }^{6}$ were completed. Free flow rate tests were attempted, and urine analysis was undertaken before urodynamic testing in each case. Findings were recorded contemporaneously on an electronic urodynamic database. Urodynamic testing was performed in accordance with the recommendations of the International Continence Society ${ }^{7}$. A rectal line was used to measure abdominal pressure and 2 lines were passed into the bladder; an $8 \mathrm{Ch}$ catheter was used for filling and a very fine tube (16G- the type of catheter used for epidural analgesia administration) to measure $P_{\text {ves }}$. The use of the two separate tubes enabled recording of the urethra pressure profile, which was undertaken in the supine position. The filling cystometry was then undertaken while the patients were standing next to a flow meter, with a filling rate ranging between 30 and $50 \mathrm{~mL} / \mathrm{min}$ using radiological contrast at room temperature. Video urodynamic screening during filling cystometry was undertaken with a $\mathrm{C}$-arm image intensifier, to look for contrast escape past the sphincter [SUI; Figure 1] and for vesicoureteric reflux (VUR). 
The decision to use a penile clamp was made by the clinician after commencement of the filling cystometry for each individual. Clamp application was considered if it became clear that leakage was so severe that bladder filling was no longer occurring, precluding accurate identification of the incontinence mechanism and/ or reliable recording of $\mathrm{P}_{\text {ves }} /$ bladder volume. Applying the clamp was undertaken to avoid having to abandon the test. Thus clamp use allowed completion of tests affected by severe incontinence during the early stages of filling cystometry (where leakage occurred at a rate equivalent to the filling rate), which would otherwise not have been completed. The device used was the Thomson Walker penile compression clamp [Figure 1]. At cystometric capacity, filling was stopped and the patient was prepared for pressure flow studies (PFS). Once the patient was in position adjacent to the flowmeter, the clamp was removed by the urodynamicist, synchronous with giving permission to void.

Urodynamic findings and diagnosis were derived based on the International Continence Society recommendations ${ }^{8}$. Urodynamic parameters were derived and checked for plausibility, removing the effects of any artefacts.

\section{Data extraction and statistical analysis}

Statistical comparisons were undertaken using the Independent t-test with significance inferred at $p<0.05$.

\section{Results}

166 men with PPI were identified, each presenting with symptoms of incontinence from the time of surgery, for whom urodynamic testing was undertaken. A penile clamp was used to enable completion of video-urodynamics for 30 of these men, and to avoid having to abandon the test. The mechanism of incontinence was identified prior to clamp application. In all 30, it was then possible to stop the leakage and thereby achieve filling to cystometric capacity.

Overall mean age was 67 years (46-86 years), and there was no significant age difference between those men for whom a penile clamp was used compared with those where it was not [Table 1]. General assessment parameters comparing the clamp-facilitated subgroup and the overall study population are presented in Table 1. Free flow rate testing [Table 1] identified the mean maximum free flow rate $\left(Q_{\max }\right)$ for the clamp subgroup was $14.7 \mathrm{ml} / \mathrm{s}$ and mean voided volume was $98 \mathrm{mls}$, similar to the overall PPI population [Table 1]. Bladder diaries, where completed, showed a low maximum voided volume in the clamp subgroup. For the ICIQMLUTS score the clamp subgroup reported a higher frequency of incontinence score and impact on quality of life [Table 1].

Urodynamic parameters are presented in Table 2. Urethral pressure profilometry identified that the mean maximum urethral closure pressure (MUCP) was low in the clamp subgroup (31.6 $\mathrm{cmH}_{2} \mathrm{O}$, compared with 46.5 for the overall PPI population; $p<0.001$ ) [Table 2]. Application of the clamp did not alter the subsequent vesical pressure recording, or the 
calculated detrusor pressure; minimal artefact was seen in the line when the clamp was applied, and often there was no apparent artefact generated by clamp application [Figure 1]. The average volume at strong desire to void and cystometric capacity were lower in the clamp subgroup [Table 2]. The mean cystometric capacity (CC) for the no-clamp group was $328 \mathrm{mls}$, which was significantly higher than the $\mathrm{CC}$ in men requiring a clamp (mean $225 \mathrm{mls} ; p<0.001$ ). Presence of DO was more easily identified in individual cases with severe incontinence when the clamp was applied [Figure 2]. The difference in the average resting detrusor pressure at capacity in both groups was not statistically significant. Video urodynamic screening identified only one PPI patient with VUR, and this person was not in the clamp subgroup.

Flow rates during PFS were comparable between the clamp subgroup and the PPI population, but the mean $P_{\text {det }} Q_{\max }$ was lower in the clamp subgroup [Table 2]. This may have been because there was insufficient time for a detrusor contraction to develop before complete leakage for some men. Post void residual volumes were small $(24 \mathrm{ml}$ for the no-clamp group, $6 \mathrm{ml}$ for the clamp group; $p=0.302$ ) [Table 2]

The final urodynamic diagnoses are presented in Table 3. For the clamp subgroup, the diagnosis was: urodynamic stress incontinence (USI) for 10/ 30 (33\%); detrusor overactivity incontinence (DOI) for 1/ 30 (3\%); and, mixed urinary incontinence (USI plus DOI) for 19/ 30 (63\%). All men were able to tolerate the presence of the penile clamp to enable completion of the urodynamic test as there were no reports on the database of any premature removal of the clamps and none related to pain or discomfort.

\section{Discussion}

The current study describes a practical approach to delivering a more complete urodynamic evaluation of men with severe post-surgical incontinence. For men with severe SUI, a penile clamp can be applied if severe leakage develops at any time during the progress of filling cystometry. The use of a penile clamp enabled additional filling of the bladder to provide a clearer interpretation of cystometric capacity and delivered the ability to undertake PFS. Use of the clamp also allowed clearer identification of DO. The clamp was well tolerated in the context of the short duration of use for the study. Applying and removing the clamp did not generate undue artefact in the vesical and detrusor pressure traces, and it did not affect the ability of the filling pump to instil contrast into the bladder. PFS, however, remain technically difficult as patients with external urethral sphincter (EUS) failure are at risk of experiencing total incontinence the instant the clamp is removed, as opposed to voluntarily generating detrusor contraction and sphincter relaxation.

We combined the pressure recording with videourodynamic imaging. This was able to establish that VUR was not a feature unmasked by placement of the penile clamp, indicating no evidence that the clamp was associated with increased upper urinary tract pressures, or reflux of potentially infected urine. Penile clamps in various designs are used for incontinence management but some practitioners have suggested they could potentially be unsafe for use by men with storage symptoms. We did not demonstrate VUR in anyone, including men with 
detrusor overactivity (20 of the 30 men in the clamp subgroup) and therefore did not find evidence to support this safety concern. Videourodynamic screening also made it possible to identify USI with a penile clamp in position, as the location of both the EUS and the clamp are clearly visible; accordingly, intrusion of contrast into this area in the presence of raised abdominal pressure and stable detrusor pressure whilst screening provides a clear demonstration of sphincter insufficiency.

In the subgroup for whom a clamp was felt necessary by the investigator, incontinence severity appeared to be worse than for the overall PPI population. This can be surmised from the MUCP, which was significantly lower. Likewise, the group using the clamp gave higher scores for incontinence severity ("How often do you leak urine"), and interference with everyday life. Some urodynamic parameters were significantly different, including the bladder volume at first desire and strong desire to void, the cystometric capacity, and the detrusor pressure at maximum flow, which were significantly lower in the clamp group.

The limitations of the study relate to the incomplete data for the range of observations made, in particular the symptom scores and bladder diaries, and some of the urodynamic parameters. This in part reflects real life experience of the difficulties patients have completing such scores when living with severe incontinence. In addition, a healthcare professional undertaking a urodynamic test may elect not to undertake urethral pressure profilometry if the individual patient's urethra is reported to be uncomfortable or is found to be difficult to catheterise. The urethral profile length in the clamp subgroup paradoxically was longer than for the PPI population overall; we think this is probably due to a lack of a clear sphincter peak on some of the profiles, making it hard for the practitioner to establish the closure-profile start and end points, and leading to an over-estimate of the functional length.

In conclusion, this study shows that the use of a penile clamp during urodynamics for incontinent men who have had a radical prostatectomy can optimise the test by aiding additional bladder filling in selected patients. This allows for a clearer interpretation of cystometric capacity and ability to undertake PFS. The short-term use in this context is well tolerated and does not raise any safety concerns.

\section{Disclaimer}

No conflict of interest to be declared.

\section{References}

1. Comiter CV, Dobberfuhl AD: The artificial urinary sphincter and male sling for postprostatectomy incontinence: Which patient should get which procedure? Investigative and clinical urology 2016, 57(1):3-13.

2. Solomon E, Veeratterapillay R, Malde S, Harding C, Jillian Greenwell T: Can filling phase urodynamic parameters predict the success of the bulbar artificial urinary sphincter in treating post-prostatectomy incontinence?; 2016.

3. Jura $\mathrm{YH}, \mathrm{Comiter} \mathrm{CV}$ : Urodynamics for postprostatectomy incontinence: when are they helpful and how do we use them? Urol Clin North Am 2014, 41(3):419-427, viii. 
4. Huckabay C, Twiss C, Berger A, Nitti VW: A urodynamics protocol to optimally assess men with post-prostatectomy incontinence. Neurourol Urodyn 2005, 24(7):622-626.

5. Hillman BJ: Evaluation of postsurgical urethra and urethral strictures by meatal compression-voiding urethrography. Urology 1981, 17(1):95-98.

6. Bright $E$, Cotterill N, Drake M, Abrams P: Developing and validating the International Consultation on Incontinence Questionnaire bladder diary. Eur Urol 2014, 66(2):294300.

7. Schafer W, Abrams P, Liao L, Mattiasson A, Pesce F, Spangberg A, Sterling AM, Zinner NR, van Kerrebroeck P, International Continence S: Good urodynamic practices:

uroflowmetry, filling cystometry, and pressure-flow studies. Neurourol Urodyn 2002, 21(3):261-274.

8. Abrams $P$, Cardozo L, Fall M, Griffiths D, Rosier P, Ulmsten U, Van Kerrebroeck P, Victor A, Wein A, Standardisation Sub-Committee of the International Continence S: The standardisation of terminology in lower urinary tract function: report from the standardisation sub-committee of the International Continence Society. Urology 2003, 61(1):37-49. 


\section{Figure legends:}

Figure 1: A: Thomson Walker Penile Clamp. B: Image from a video urodynamic study of a patient who previously had a radical prostatectomy, showing bladder filled with contrast, and contrast in the proximal, membranous and bulbar urethra down to the level of a penile clamp. C: Section of urodynamic recording during filling cystometry illustrating the moment a penile clamp is applied, showing the lack of effect on vesical (blue) and detrusor (green; inset shows a close up of the lack of visible effect on detrusor pressure at the exact moment of application).

Figure 2. Conclusion of filling cystometry and the pressure flow study in a man who previously had radical prostatectomy. The patient was in the supine position and was leaking continuously. Detrusor overactivity was suspected but could not be identified with certainty. Thus, to facilitate pressure recording, a penile clamp was applied (1). At 2 , the man was helped from the supine to the standing position. Detrusor overactivity then became clearly evident (3). The penile clamp was then removed (4) to allow pressure flow recording. 
Tables:

Table 1: Population characteristics.

\begin{tabular}{|c|c|c|c|c|}
\hline \multicolumn{5}{|l|}{ Population characteristics } \\
\hline & All & Clamp not needed & Clamp & \\
\hline Number & 166 & 136 & 30 & \\
\hline Age (years) & $67.0(46-86 ; 7.2)$ & $66.8(46-86 ; 7.5)$ & $67.4(49-76 ; 5.6)$ & \\
\hline \multicolumn{4}{|l|}{ Free Flowmetry } & $P$ Value $^{t}$ \\
\hline Recorded & 121 & 106 & 15 & \\
\hline Voided Volume (ml) & $193(10-940 ; 183.6)$ & 206 (10-940; 193.2) & $98(22-292 ; 70.9)$ & $p=0.034$ \\
\hline Qmax (ml/s) & $19.4(1-51 ; 11.2)$ & $20.1(1-51 ; 11.4)$ & $14.7(5-31 ; 8.4)$ & $p=0.083$ \\
\hline \multicolumn{5}{|l|}{ Bladder Diary } \\
\hline Completed & 108 & 96 & 12 & \\
\hline Maximum voided volume (ml) & $467(100-1200 ; 209.6)$ & $475(100-1200 ; 207)$ & $405(120-1000 ; 220)$ & $\mathrm{p}=0.007$ \\
\hline Average voided volume (ml) & $232(50-647 ; 103.8)$ & $235(50-647 ; 106.6)$ & $212(100-350 ; 70.7)$ & $p=0.525$ \\
\hline Total voided volume (ml) & $1803(653-4530 ; 717.8)$ & $1812(653-4530 ; 735)$ & $1714(750-2500 ; 501.5)$ & $p=0.700$ \\
\hline \multicolumn{5}{|l|}{ ICIQ - MLUTS Questionnaire } \\
\hline Completed & 120 & 102 & 18 & \\
\hline How often do you leak urine $\ddagger$ & $4.1(0-5 ; 1.1)$ & $4.0(0-5 ; 1.2)$ & $4.8(4-5 ; 0.4)$ & $\mathrm{p}=0.005$ \\
\hline Incontinence bother Score§ & $9.0(4-10 ; 1.7)$ & $8.9(4-10 ; 1.8)$ & $9.6(7-10 ; 0.8)$ & $p=0.097$ \\
\hline Interference with everyday§ & $8.7(0-10 ; 1.9)$ & $8.5(0-10 ; 2)$ & $9.5(7-10 ; 0.9)$ & $p=0.048$ \\
\hline
\end{tabular}

Values presented as mean (range; SD). Clamp: the parameters observed with the clamp in situ for those patients in whom the device was used. $¥$ Score out 5 , where 5 is severe. $§ S c o r e$ out of 10, where 10 is severe. $S D=S t a n d a r d$ deviation. NR=not recorded. + Independent $t$-test. 
Table 2: Urodynamic findings.

\begin{tabular}{|l|l|l|l|l|}
\hline & All Patients & Clamp not needed & Clamp & $P_{\text {Value }}{ }^{+}$ \\
\hline MUCP (cmH2O) & $46.5(0-100 ; 20.6) \mathrm{n}=140$ & $50.2(15-100 ; 19.1) \mathrm{n}=112$ & $31.6(0-87 ; 19.3) \mathrm{n}=28$ & $\mathrm{P}<0.001$ \\
\hline Profile length (cm) & $1.3(0-8 ; 1.6) \mathrm{n}=25$ & $0.8(0.3-1.5 ; 0.38) \mathrm{n}=18$ & $2.36(0-8 ; 2.7) \mathrm{n}=7$ & $\mathrm{p}=0.033$ \\
\hline First Desire to Void (ml) & $193.2(0-527 ; 108.1) \mathrm{n}=135$ & $205.5(0-527 ; 107.8) \mathrm{n}=111$ & $136.1(25-377 ; 89.3) \mathrm{n}=24$ & $\mathrm{P}=0.004$ \\
\hline Normal Desire to Void & $286(80-499 ; 118.3) \mathrm{n}=44$ & $304(100-499 ; 113.3) \mathrm{n}=34$ & $226.8(80-497 ; 114.8) \mathrm{n}=10$ & $\mathrm{p}=0.068$ \\
\hline Strong Desire to Void (ml) & $242(20-580 ; 122) \mathrm{n}=44$ & $267(58-580 ; 118.7) \mathrm{n}=36$ & $132(20-217 ; 62.1) \mathrm{n}=8$ & $\mathrm{p}=0.003$ \\
\hline Cystometric Capacity (ml) & $310(54-708 ; 131.5) \mathrm{n}=162$ & $328(54-708 ; 130.2) \mathrm{n}=133$ & $226(86-500 ; 102.3) \mathrm{n}=29$ & $\mathrm{p}<0.001$ \\
\hline Vesicoureteric Reflux & 1 & 1 & 0 & - \\
\hline $\begin{array}{l}\text { Resting detrusor pressure } \\
\text { at capacity (cmH20) }\end{array}$ & $3.7(0-39 ; 4.8) \mathrm{n}=162$ & $3.6(0-39 ; 4.8 .6) \mathrm{n}=132$ & $4.1(0-15 ; 4.8) \mathrm{n}=30$ & $\mathrm{P}=0.526$ \\
\hline Qmax during PFS (ml/s) & $14.5(2-42 ; 7.5) \mathrm{n}=149$ & $14.6(2-42 ; 7.8) \mathrm{n}=125$ & $13.9 *(4-24 ; 5.5) \mathrm{n}=24$ & $\mathrm{p}=0.650$ \\
\hline PdetQmax (cmH2O) & $21.9(0-168 ; 20.4) \mathrm{n}=126$ & $23.9(0-168 ; 20.4) \mathrm{n}=106$ & $11.0(0-58 ; 15.5) \mathrm{n}=20$ & $\mathrm{p}=0.009$ \\
\hline Voided Volume (ml) & $294(53-708 ; 130.8) \mathrm{n}=147$ & $303.9(54-708 ; 132.4) \mathrm{n}=124$ & $241(53-500 ; 103.3) \mathrm{n}=23$ & $\mathrm{p}=0.034$ \\
\hline Residual Volume (ml) & $21.2(0-500 ; 72.2) \mathrm{n}=140$ & $23.8(0-500 ; 77.5) \mathrm{n}=120$ & $5.7(0-64 ; 15.8) \mathrm{n}=20$ & $\mathrm{p}=0.302$ \\
\hline
\end{tabular}

Values presented as mean (range; SD). Clamp: the parameters observed with the clamp in situ for those patients in whom the device was used. Clamp: the filling cystometry parameters observed with the clamp in situ: for pressure flow studies, the clamp was removed beforehand. $M U C P=$ maximum urethral closure pressure. $N R=$ Not recorded. $S D=$ standard deviation. + Independent t-test.

Table 3: Final urodynamic diagnosis for all patients.

\begin{tabular}{|l|l|l|l|}
\hline Diagnosis & \multicolumn{3}{|l|}{} \\
\hline & All & Clamp not needed & Clamp \\
\hline Normal & 10 & 10 & 0 \\
\hline Urodynamic Stress Incontinence & 63 & 53 & 10 \\
\hline Detrusor Overactivity Incontinence & 25 & 24 & 1 \\
\hline Mixed urinary incontinence & 67 & 48 & 19 \\
\hline Reduced detrusor compliance & 1 & 1 & 0 \\
\hline
\end{tabular}




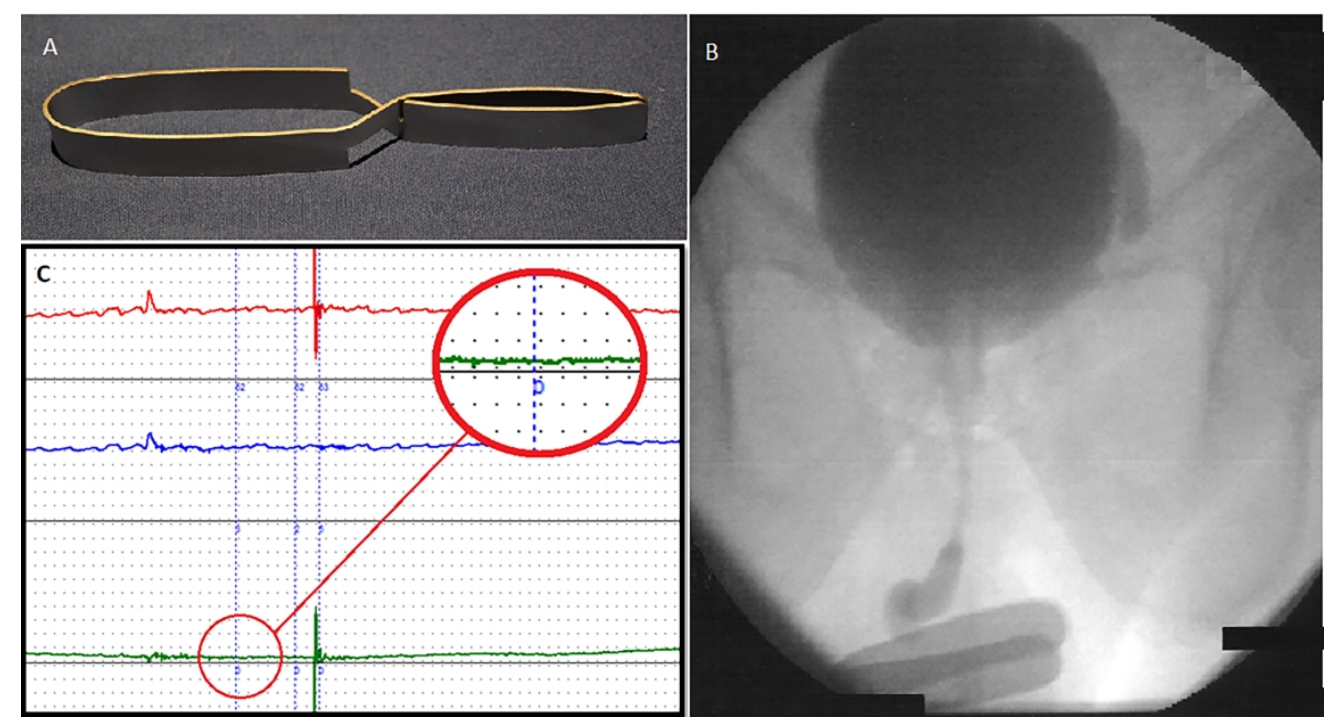

Figure 1: A: Thomson Walker Penile Clamp. B: Image from a video urodynamic study of a patient who previously had a radical prostatectomy, showing bladder filled with contrast, and contrast in the proximal, membranous and bulbar urethra down to the level of a penile clamp. C: Section of urodynamic recording during filling cystometry illustrating the moment a penile clamp is applied, showing the lack of effect on vesical (blue) and detrusor (green; inset shows a close up of the lack of visible effect on detrusor pressure at the exact moment of application).

$271 \times 146 \mathrm{~mm}(300 \times 300 \mathrm{DPI})$ 


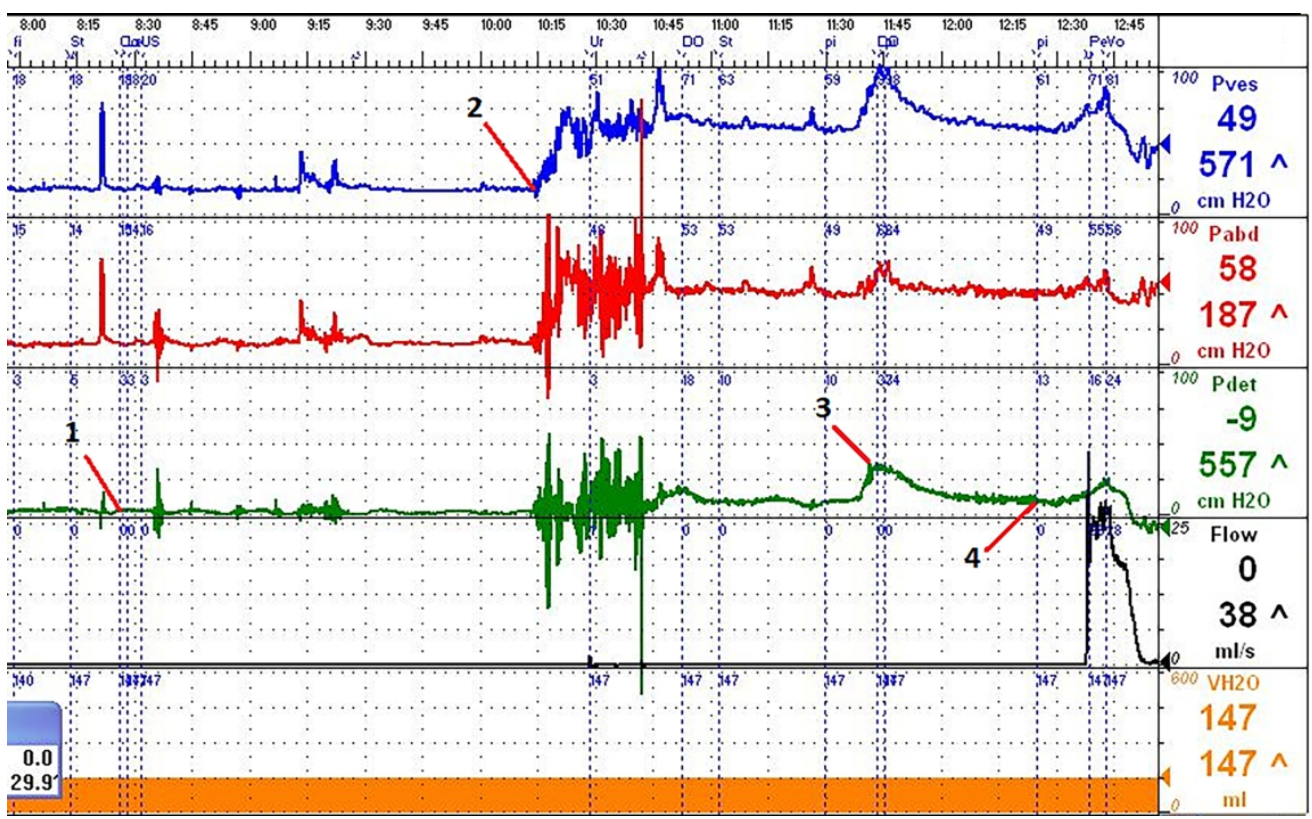

Figure 2. Conclusion of filling cystometry and the pressure flow study in a man who previously had radical prostatectomy. The patient was in the supine position and was leaking continuously. Detrusor overactivity was suspected, but could not be identified with certainty. Thus, to facilitate pressure recording, a penile clamp was applied (1). At 2, the man was helped from the supine to the standing position. Detrusor overactivity then became clearly evident (3). The penile clamp was then removed (4) to allow pressure flow recording. 